\title{
Tyrosine Phosphorylation of Netrin Receptors in Netrin-1 Signaling
}

\author{
Xiu-Rong Ren ${ }^{b}$ Yan Hong ${ }^{b}$ Zhu Feng ${ }^{a}$ Hong-Mei Yang ${ }^{b}$ Lin Mei $^{a}$ \\ Wen-Cheng Xiong ${ }^{\text {a }}$ \\ aProgram of Developmental Neurobiology, Institute of Molecular Medicine and Genetics and Department of \\ Neurology, Medical College of Georgia, Augusta, Ga., and b Department of Pathology, University of Alabama at \\ Birmingham, Birmingham, Ala., USA
}

\section{Key Words}

Netrin-1 - Deleted in colorectal cancer · Neogenin · Focal adhesion kinase $\cdot$ Fyn

\begin{abstract}
Deleted in colorectal cancer (DCC) and neogenin are receptors of netrins, a family of guidance cues that promote axon outgrowth and guide growth cones in developing nervous system. The intracellular mechanisms of netrins, however, remain elusive. In this paper, we show that both DCC and neogenin become tyrosine phosphorylated in cortical neurons in response to netrin-1. Using a site-specific antiphosphor DCC antibody, we show that Y1420 phosphorylation is increased in netrin-1-stimulated neurons and that tyrosinephosphorylated DCC is located in growth cones. In addition, we show that tyrosine-phosphorylated DCC selectively interacts with the Src family kinases Fyn and Lck, but not Src, c-Abl, Grb2, SHIP1, Shc, or tensin, suggesting a role of Fyn or Lck in netrin-1-DCC signaling. Of interest to note is that tyrosine-phosphorylated neogenin and uncoordinated $5 \mathrm{H} 2(\mathrm{Unc} 5 \mathrm{H} 2)$ not only bind to the Src homology $2(\mathrm{SH} 2)$ domains of Fyn and SHP2, but also interact with the $\mathrm{SH} 2$ domain of SHIP1, suggesting a differential signaling between DCC and neogenin/Unc5H2. Furthermore, we demonstrate that inhibition of Src family kinase activity attenu-
\end{abstract}

ated netrin-1-induced neurite outgrowth. Together, these results suggest a role of Src family kinases and tyrosine phosphorylation of netrin-1 receptors in regulating netrin-1 function.

Copyright $\odot 2008$ S. Karger AG, Basel

\section{Introduction}

Axon outgrowth and guidance are essential for the establishment of neuronal connections during embryogenesis and nerve regeneration in injured brains. $\mathrm{Ne}$ trins, a family of conserved secreted molecules, play important roles in promoting axon outgrowth, guiding neuronal growth cones, and regulating neuronal branching in the developing nervous system [1-4]. They are capable of not only attracting some axons, but also repelling others $[3,5]$. Two classes of receptors, deleted in colorectal cancer (DCC) and uncoordinated 5 (UNC-5), are known to mediate netrins' functions. The DCC family receptors including DCC and neogenin in vertebrates [6], UNC-40 in Caenorhabditis elegans [7], and Frazzled in Drosophila $[8,9]$. DCC and UNC-40 are required for growth cone attraction by netrins [10]. UNC-5, on the other hand, appears to mediate netrin's repulsive effect [11-15]. The exact role of neogenin in netrin-1 functions

Tel. +1 706721 5148, Fax +1 706721 8685, E-Mail wxiong@mcg.edu 
is unclear. It is of interest to note that neogenin is shown to be a receptor of repulsive guidance molecule (RGM), a GPI-liked cell-surface protein implicated in repulsive growth cone guidance $[16,17]$, suggesting that it may play a different role in axon guidance. The intracellular mechanisms downstream of DCC and neogenin remain largely unknown.

Tyrosine phosphorylation has been implicated in axonal outgrowth and guidance induced by several extracellular guidance cues. In response to ephrins, Eph receptor tyrosine kinases become activated. Tyrosine kinase activity of Eph receptors is required for their function in controlling axon guidance in developing brain [18]. Slit receptor robo that mediates the repulsive response can be tyrosine phosphorylated by the Abl tyrosine kinase, which attenuates slit responses [19]. Several lines of evidence demonstrate the importance of tyrosine phosphorylation in netrin-1-mediated axonal pathfinding. UNC40, the DCC homologue in C. elegans, and UNC-5 are tyrosine phosphorylated in vitro and in C. elegans [20]. UNC-5 tyrosine phosphorylation appears to be necessary for netrin-1 function in C. elegans [21]. CLR-1, a transmembrane receptor tyrosine phosphatase, appears to be a negative regulator of the UNC-40-mediated attractive response in C. elegans [22]. Interestingly, recent publications suggest that focal adhesion kinase (FAK), a major cell adhesion activated tyrosine kinase, appears to be a positive regulator of DCC tyrosine phosphorylation, and DCC-mediated neurite outgrowth and attractive growth cone turning [23-25]. While DCC tyrosine phosphorylation has been implicated in netrin-1-induced axon pathfinding $[25,26]$, exactly how DCC tyrosine phosphorylation participates and the role of neogenin tyrosine phosphorylation in netrin-1 signaling remain largely unclear.

In this paper, we show that DCC and neogenin are tyrosine phosphorylated in rat cortical neurons in response to netrin-1 stimulation. Phosphorylated DCC, neogenin, and uncoordinated $5 \mathrm{H} 2$ (Unc5H2) interact subsequently with the Src homology 2 (SH2) domain containing signaling proteins including Fyn and Lck. In addition, phosphorylated neogenin/Unc5H2, but not DCC, binds to the SH2 domain of SHIP1. Inhibition of Src family kinases abolished netrin-1-stimulated DCC tyrosine phosphorylation and neurite outgrowth response in rat cortical explants. These results suggest a differential signaling between DCC and neogenin, and demonstrate a role of an Src family kinase in phosphorylating DCC and mediating netrin-1 function.

\section{Experimental Procedures}

Reagents

To generate antibodies specific for phospho-Y1420 in DCC, rabbit antiserum was raised against the phosphopeptide TEDSANVYpEQDDLSE (residues of 1,413-1,427 of human DCC with the addition of a cysteine at the $\mathrm{N}$-terminus). The serum was passed through a column of the cognate nonphosphopeptide, and the antibody was purified by affinity chromatography with the phosphopeptide column. Rabbit polyclonal anti-neogenin antibodies were generated using glutathione-S-transferase (GST)neogenin (residues of 1,158 to 1,527 of mouse neogenin) as an antigen. Monoclonal antibodies were purchased from Santa Cruz Biotechnology (Santa Cruz, Calif., USA; anti-Myc), Sigma Chemical Co. (St. Louis, Mo., USA; anti-Flag), Oncogene Research Products, Inc. (Cambridge, Mass., USA; anti-DCC), and Transduction Labs (Lexington, Ky., USA; anti-FAK, and RC20). Polyclonal anti-DCC antibodies were purchased from Santa Cruz Biotechnology (Santa Cruz, Calif., USA; A20).

Stable HEK 293 cells expressing human netrin-1 were provided by J.Y. Wu (Washington University) [27]. Unless otherwise indicated, condition medium containing $\sim 200 \mathrm{ng} / \mathrm{ml}$ human netrin-1 was used for stimulation.

PP1 (4-amino-5-(4-methylphenyl)-7-(t-butyl)pyrazolo[3,4-d]pyrimidine),PP2(4-amino-5-(4-chlorophenyl)-7-(t-butyl)pyrazolo[3,4-d]pyrimidine), and PP3 (4-amino-7-phenylpyrazol[3,4-d]pyrimidine) were purchased from Calbiochem (San Diego, Calif., USA).

\section{Expression Vectors}

cDNAs encoding neogenin, UNC-5h2, DCC, or DCC mutants were amplified by PCR and subcloned into mammalian expression vectors downstream of a signal peptide and a Flag or a Myc epitope tag under the control of the CMV promoter [24]. FAK constructs were described previously [29]. Point mutations in DCC were generated using the quick change kit (Stratagene). The authenticity of all mutants was verified by DNA sequencing.

HEK 293 Cell Culture and Transfection

HEK 293 cells were maintained in DMEM supplemented with $10 \%$ fetal calf serum, $100 \mathrm{U} / \mathrm{ml}$ penicillin $\mathrm{G}$ and streptomycin (Gibco). Cells were plated at a density of $10^{6}$ cells per $10-\mathrm{cm}$ culture dish for $12 \mathrm{~h}$ before transfection using the calcium phosphate precipitation method. Thirty-six hours after transfection, cells were lysed in the modified RIPA buffer (50 mM Tris- $\mathrm{HCl}, \mathrm{pH} 7.4$, $150 \mathrm{~mm}$ sodium chloride, $1 \%$ NP40, $0.25 \%$ sodium deoxycholate, and proteinase inhibitors) [30]. Lysates were subjected to immunoprecipitation or immunoblotting.

\section{Immunoprecipitation}

Immunoprecipitation was carried out as previously described [30]. Briefly, cell lysates (1 mg of protein) were incubated with indicated antibodies $(1-2 \mu \mathrm{g})$ at $4^{\circ} \mathrm{C}$ for $1 \mathrm{~h}$ in a final volume of $1 \mathrm{ml}$ modified RIPA buffer with constant rocking. After the addition of protein A/G-agarose beads, the reaction was incubated at $4{ }^{\circ} \mathrm{C}$ for $1 \mathrm{~h}$. Immune complexes were resolved by SDS-PAGE and subjected to immunoblotting. 
Yeast Two-Hybrid Studies

The C-terminal domain of chicken FAK (amino acid residues $800-1,052)$ was generated by PCR, subcloned downstream of the Gal4 DNA-binding domain (BD) in pGBT10 as described previously [24]. Neogenin, neogenin mutants, and DCC C-terminal tail were subcloned downstream of the GAL-4 DNA activation domain $(\mathrm{AD})$ in pGAD424. Yeast vectors were cotransformed into Y190. Interactions were characterized by growth of transformed yeasts on plates without leucine, tryptophan, or histidine, and by both a filter assay and liquid assay for $\beta$-galactosidase activity as described previously [30].

\section{GST Pull-Down Assay}

GST pull down assay was carried out as described previously [30]. Briefly, transiently transfected HEK 293 cells were lysed in the modified RIPA buffer. Cell lysates were precleared with GST immobilized on glutathione-Sepharose 4B (Pharmacia, Piscataway, N.J., USA), and then incubated with indicated GST fusion proteins $(2-5 \mu \mathrm{g})$ immobilized on glutathione-Sepharose beads at $4^{\circ} \mathrm{C}$ for $1 \mathrm{~h}$ with constant rocking. Beads were washed three times with the modified RIPA buffer, and bound proteins were resolved by SDS-PAGE and subjected to immunoblotting.

\section{Rat Cortical Neuronal Culture}

Primary cortical neurons were cultured as described previously [31]. Briefly, embryos (E17) were removed from anesthetized pregnant Sprague-Dawley rats. Cerebral cortexes were dissected out and chopped into small pieces after the meninges were removed thoroughly. After incubation in PBS solution containing $0.125 \%$ (w/v) trypsin (Sigma) for $20 \mathrm{~min}$ at $37^{\circ} \mathrm{C}$, digested tissues were mechanically triturated by repeated passages through a Pasteur pipette in PBS solution containing 0.05\% (w/v) DNase (Sigma). Dissociated cells were suspended in the neurobasal medium with B-27 supplement (Life Technologies), and $100 \mathrm{U} / \mathrm{ml}$ penicillin/streptomycin and plated on poly-D-lysine coated dishes (Corning). After 1 day of incubation at $37^{\circ} \mathrm{C}$ in a $5 \% \mathrm{CO}_{2}$ atmosphere, $10 \mu \mathrm{M}$ cytosine arabinoside was added to inhibit the proliferation of glia. In these cultures, over $95 \%$ cells are neurons with little contamination of immature neural progenitors or lineage-restricted progenitors.

\section{Explant Cultures}

Explant assays were carried out as described previously [32]. Timed pregnant rats were anesthetized, and the ensuing procedures were performed under sterile conditions. E14.5 embryos were dissected from uterine horns. The dorsolateral cortex was dissected out and cut into approximately $200 \times 200-\mu \mathrm{m}$ pieces that spanned the full thickness of the cortical wall with thin tungsten needles. Explants were embedded in a three-dimensional collagen (Roche Diagnostics, Indianapolis, Ind., USA) matrix with the ventricular side up. After polymerization, gels were covered with Ham's F-12 medium supplemented with $5 \%$ heat-inactivated horse serum (Gibco) and $100 \mathrm{U} / \mathrm{ml}$ penicillin/streptomycin, then were incubated at $37^{\circ} \mathrm{C}$ in a $5 \% \mathrm{CO}_{2}$ atmosphere. In some experiments, cortical explants were cocultured with aggregates of either netrin-1 secreting 293 cells or control 293 cells as described previously [33, 34]. Briefly, aggregates of 293 cells were cocultured with cortical explants at a distance in collagen gels. Neurite outgrowth was analyzed after $24 \mathrm{~h}$ in culture. The total neurite length for each explant was obtained by adding the lengths of all neurites (regardless of bundle thickness). Significance was assessed using Student's t test.

Tyrosine Phosphorylation of Netrin Receptors

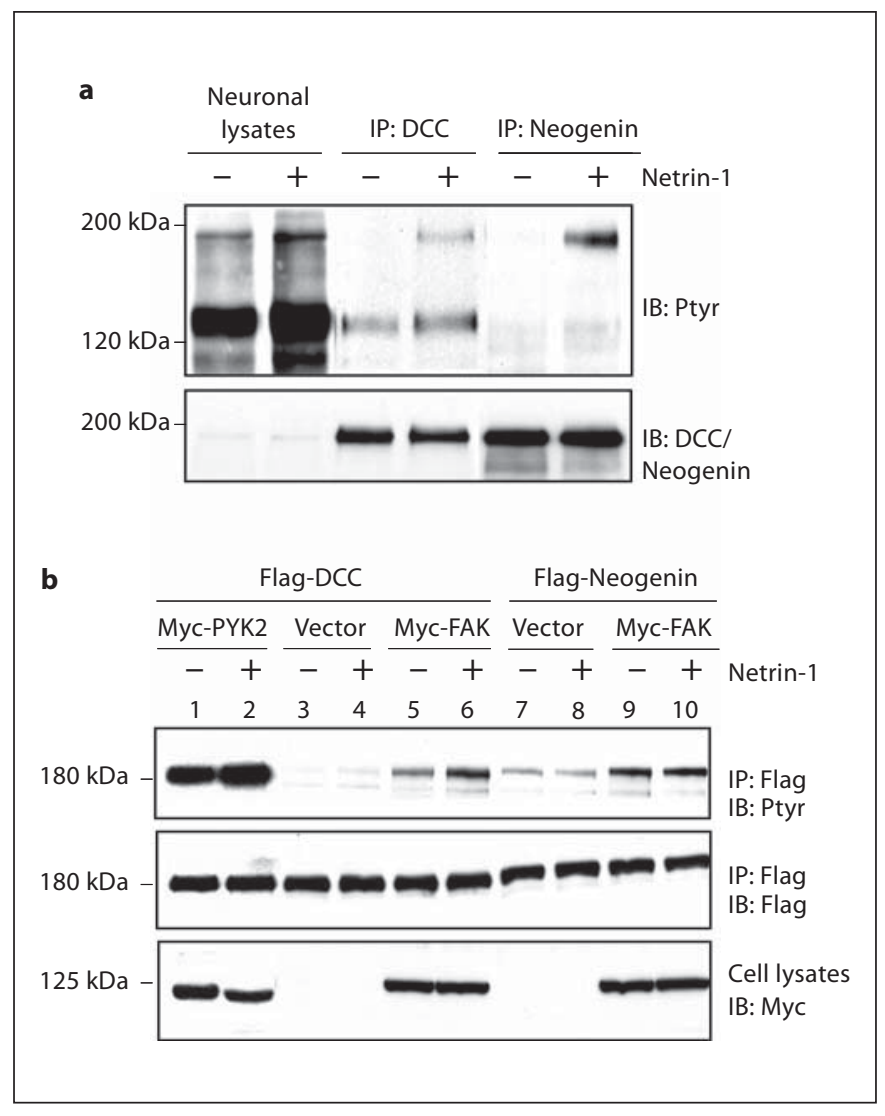

Fig. 1. Differential induction of tyrosine phosphorylation of DCC and neogenin by netrin-1. a Tyrosine phosphorylation of DCC and neogenin in netrin-1-stimulated rat cortical neurons (E17, DIV3). Neuronal lysates (500 $\mu$ g of protein) were incubated with anti-DCC or neogenin and immunoprecipitated DCC or neogenin was subjected to Western blot using the antibody RC20 and anti-DCC or neogenin (as control). b Netrin-1 induction of tyrosine phosphorylation of DCC, but not neogenin, in HEK 293 cells expressing FAK. HEK 293 cells were co-transfected Myc-FAK or PYK2 with Flag-DCC, or neogenin, and stimulated with netrin-1 for $30 \mathrm{~min}$. Cell lysates were incubated with an anti-Flag antibody for immunoprecipitation and immunoprecipitated proteins were probed with indicated antibodies.

\section{Results}

\section{Netrin-1 Induction of Tyrosine Phosphorylation of DCC and Neogenin}

To further characterize DCC and neogenin tyrosine phosphorylation in response to netrin-1, neurons were stimulated with netrin-1 and resulting lysates were analyzed for DCC and neogenin tyrosine phosphorylation. Netrin-1 was able to elicit tyrosine phosphorylation of both DCC and neogenin in rat cortical neurons (fig. 1a), 


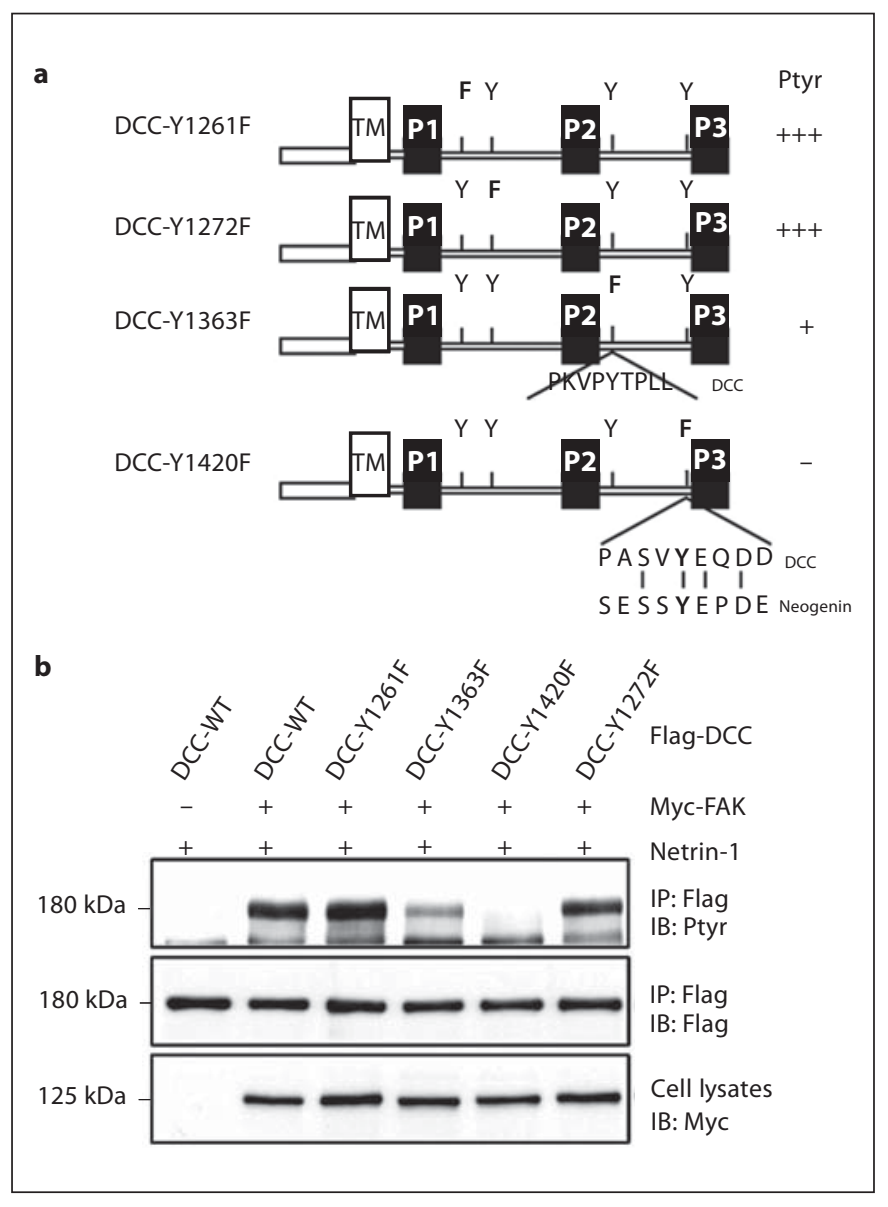

Fig. 2. Netrin-1 induction of DCC phosphorylation at Y1420 and Y1363. a Diagram of DCC tyrosine mutants. Note that only Y1420 in DCC is conserved between DCC and neogenin. b Netrin-1 induction of phosphorylation of DCC at Y1420 and Y1363 in HEK 293 cells expressing FAK. HEK 293 cells were co-transfected MycFAK with Flag-DCC or DCC tyrosine mutants, and stimulated with netrin-1 for $30 \mathrm{~min}$. Cell lysates were incubated with an antiFlag antibody for immunoprecipitation and immunoprecipitated proteins were probed with the indicated antibodies.

consistent with previous findings [24]. This event appeared to be time dependent, which peaked at $30 \mathrm{~min}$ and started to decline around 60 min upon netrin-1 stimulation (data not shown). We have recently shown that netrin-1-induced DCC tyrosine phosphorylation requires FAK signaling [24]. Here we reconstituted netrin-1 signaling in HEK 293 cells, which express a low level of FAK (data not shown), undetectable DCC, but a high level of neogenin (data not shown). Thus, they did not respond to netrin-1. Transfected Flag-DCC alone was only weakly tyrosine phosphorylated in response to netrin-1 (fig. $1 \mathrm{~b}$, lanes 3 and 4). There was a slight increase in basal tyro- sine phosphorylation of DCC when FAK was co-transfected in HEK 293 cells (fig. 1b, lane 5). However, FAK co-transfection dramatically increased DCC tyrosine phosphorylation by netrin-1 (fig. $1 \mathrm{~b}$, lane 6), in agreement with our previous finding that FAK plays a crucial role in netrin-1 induction of DCC tyrosine phosphorylation [24]. To examine the role of FAK in netrin-1 induction of neogenin tyrosine phosphorylation, FAK was cotransfected with neogenin in HEK293 cells. FAK cotransfection also caused an increase in basal neogenin tyrosine phosphorylation. However, neogenin tyrosine phosphorylation was not regulated by netrin-1 (fig. $1 \mathrm{~b}$, lanes 7-10), supporting the notion that netrin-1 was unable to stimulate or activate neogenin in non-neuronal cells and revealing a unique regulatory mechanism in neogenin tyrosine phosphorylation.

\section{Identification of Tyrosine Phosphorylation Sites in DCC}

The cytoplasmic domain of DCC contains four tyrosine residues, two in the region between $\mathrm{P} 1$ and $\mathrm{P} 2$ and another two between P2 and P3 (fig. 2a). To identify tyrosine residues that undergo phosphorylation in response to netrin-1 stimulation, we generated mutants in which an individual tyrosine residue was mutated to phenylalanine (fig. 2a). HEK 293 cells coexpressing FAK with DCC or DCC mutant were used for the identification of netrin1-induced DCC tyrosine phosphorylation site(s). FlagDCC and DCC tyrosine mutants were co-transfected into HEK 293 cells with Myc-FAK and examined for DCC tyrosine phosphorylation after netrin-1 stimulation. As shown in figure $3 \mathrm{~b}$, mutations at Y1261 or Y1272 did not appear to affect DCC tyrosine phosphorylation, suggesting little, if any, contribution from them. In contrast, mutation of Y1420 completely inhibited DCC tyrosine phosphorylation, identifying a major tyrosine phosphorylation site in DCC by netrin-1 (fig. 2b). In addition, Y1363F showed significantly reduced DCC tyrosine phosphorylation induced by netrin-1, suggesting that this residue may also be tyrosine phosphorylated (fig. 2b). It is worth noticing that cotransfection of PYK2, a FAK-related kinase, significantly increased DCC tyrosine phosphorylation even in the absence of netrin-1 (fig. 1b, lanes 1-2), and similar tyrosine residues in DCC (e.g. Y1420) were phosphorylated in response to PYK2 co-expression (data not shown). In addition, Y1420, but not Y1363, is conserved between DCC and neogenin (fig. 2a), attesting its significance in netrin-1 signaling.

To analyze the phosphorylation of DCC at Y1420 in neurons, we made a phosphorylation state-specific anti- 


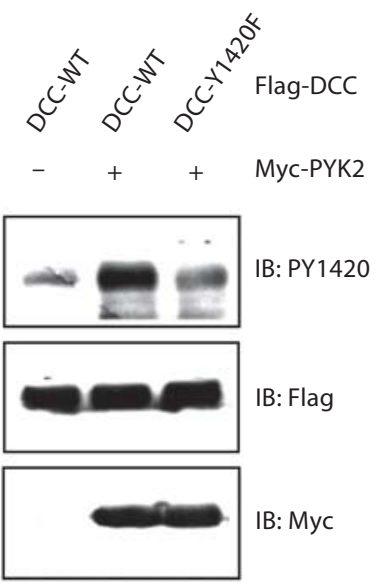

C
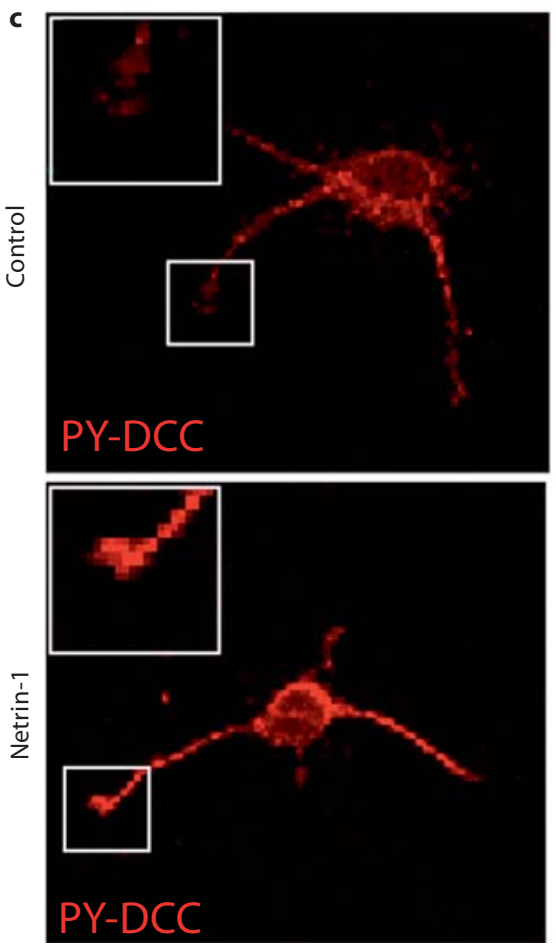

b

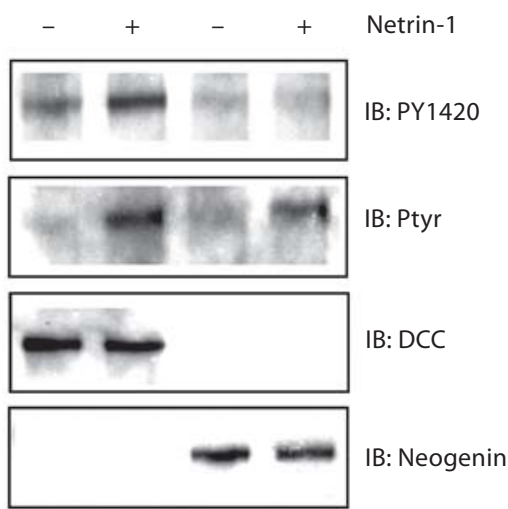

d

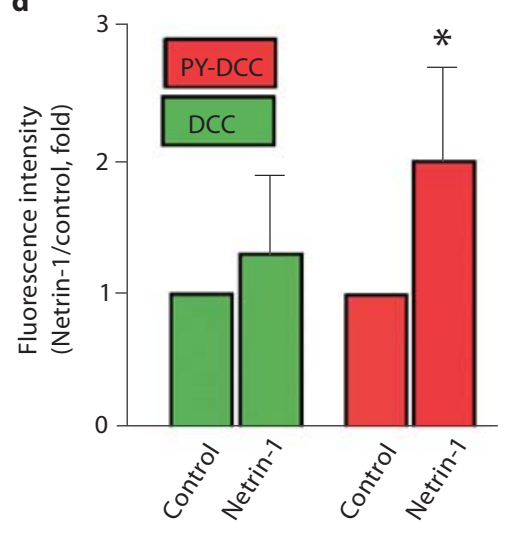

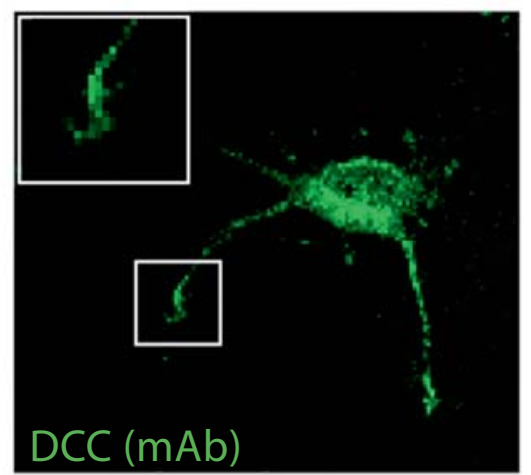
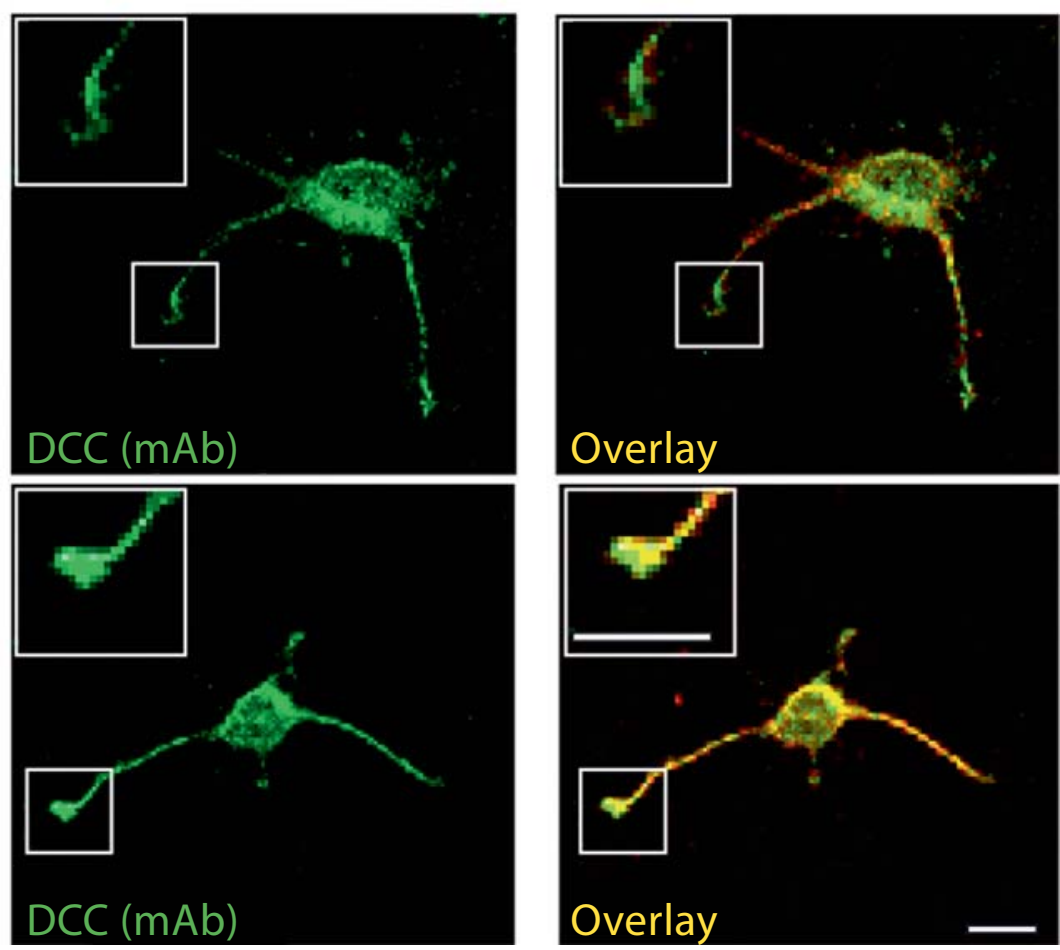

Fig. 3. Induction and localization of PY1420-DCC in neurons. a Recognition of netrin-1-induced DCC Y1420 phosphorylation in HEK 293 cells coexpressing DCC with PYK2 by PY1420 antibody. HEK 293 cells were co-transfected Myc-PYK2 with FlagDCC or DCC-Y1420F mutant. Cell lysates were subjected to immunoblotting analysis using anti-PY1420, Flag, and Myc antibodies. b Netrin-1 induction of Y1420 phosphorylation in neurons. Lysates from rat cortical neurons stimulated with or without netrin-1 for 30 min were incubated with anti-DCC or anti-neogenin antibodies for immunoprecipitation and immunoprecipated proteins were probed with indicated antibodies. c Images of immunostained DCC and tyrosine-phosphorylated DCC
(PY1420) in cortical neurons. Rat cortical neurons (E17, DIV 3) were stimulated with or without netrin-1 for $30 \mathrm{~min}$, fixed, and immunostained with anti-PY1420 antibody followed with Cy3conjugated secondary antibody and anti-DCC antibody (monoclonal) followed with FITC-conjugated secondary antibody. Immunoreactivity was visualized by a confocal fluorescence microscope. Bar $=50 \mu \mathrm{m}$. d Quantitative analyses of data in (c). Shown is an increase in immunofluorescence intensity by anti-DCC and anti-PY1420 staining per $\mu \mathrm{m}^{2}$ at the tips of neurites by netrin- 1 . Data shown are mean $\pm \mathrm{SE}(\mathrm{n}=5),{ }^{*} \mathrm{p}<0.01$, different from control neurons (t test). 
Fig. 4. Association of $\mathrm{SH} 2$ domain containing proteins with tyrosine-phosphorylated DCC. a Tyrosine-phosphorylated DCC interacts with $\mathrm{SH} 2$ domain-containing proteins in vitro in GST pull-down assays. DCC and FAK co-transfected HEK 293 cells were stimulated with netrin-1 and cell lysates were mixed with indicated GST fusion proteins immobilized on beads. Bound proteins were immunoblotted with an anti-DCC antibody. Equal amounts of GST fusion proteins were revealed by Coomassie staining (bottom). b Requirement of DCC-Y1363 and Y1420 phosphorylation for binding to the Fyn SH2 domain. HEK 293 cells were co-transfected without or with Myc-FAK and FlagDCC wild-type or tyrosine mutants, stimulated with netrin-1, and lyzed. Cell lysates were mixed with GST-Fyn-SH2 fusion proteins $(5 \mu \mathrm{g})$ immobilized on beads. Bound proteins were immunoblotted with anti-Flag antibody. Cell lysates were also probed with anti-Myc and antiFlag antibodies. a
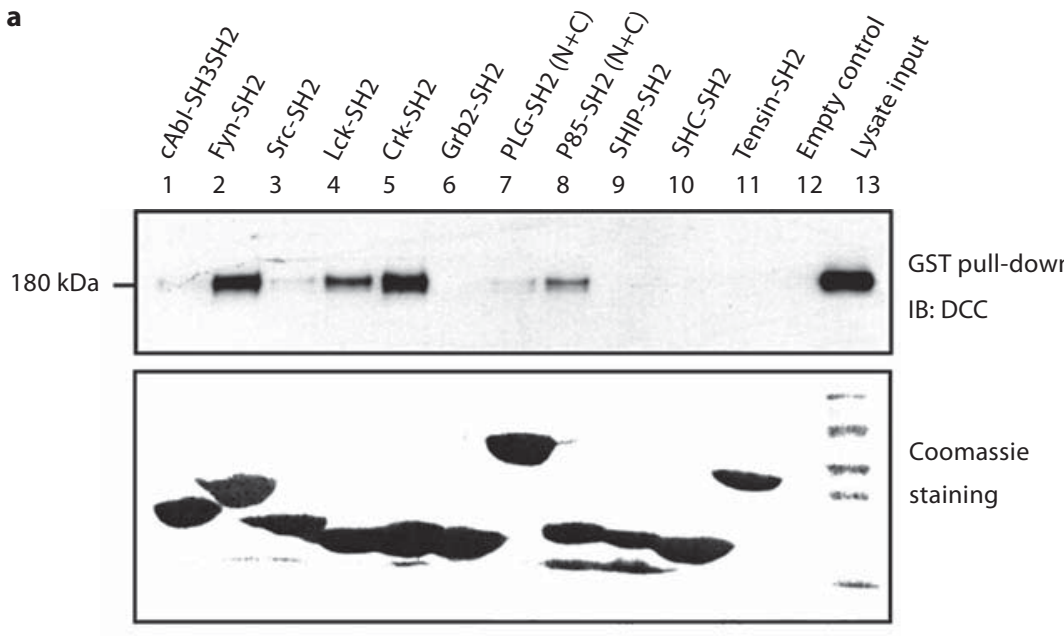

b
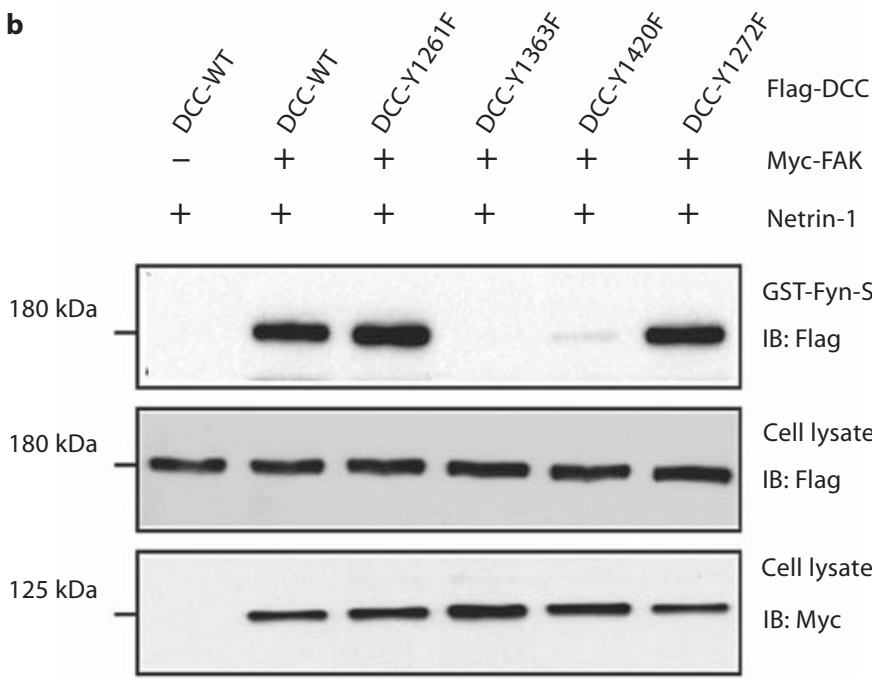

GST-Fyn-SH2

IB: Flag

Cell lysates

IB: Flag

Cell lysates

IB: Myc body. The phospho-Y1420 antibody (PY1420) recognizes wild-type DCC that has been phosphorylated, but not DCC-Y1420F or nonphosphorylated DCC (fig. 3a), indicating the specificity of the antibody to phosphorylated DCC at Y1420. We next examined whether netrin-1 is able to induce DCC phosphorylation at Y1420 in cultured neurons. As shown in figure $3 \mathrm{~b}$, while tyrosine phosphorylation of both DCC and neogenin was induced by netrin-1 (recognized by PY20), the PY1420 antibody only recognized netrin-1-induced phosphorylated DCC, but not neogenin. These results demonstrate that netrin-1 indeed stimulates phosphorylation of DCC at Y1420 in neurons in addition to the specificity of the antibody. To determine the subcellular localization of PY1420-DCC, rat cortical neurons were treated with netrin-1 for $1 \mathrm{~h}$ and stained with the PY1420 antibody. In naïve neurons, phosphorylated DCC could be detected in the soma and neurites (fig. 3c, control). Interestingly, in response to netrin-1 stimulation, there is a significant increase in PY1420 staining in growth cones (fig. 3c, d). These results support the notion that DCC tyrosine phosphorylation plays a role in neurite outgrowth and axon guidance. 
Fig. 5. Differential association of $\mathrm{SH} 2$ domain-containing proteins with tyrosinephosphorylated DCC, neogenin, and UNC5h2. a Induction of tyrosine-phosphorylation of DCC, neogenin, and UNC5h2 by co-expression of PYK2. HEK 293 cells were transiently transfected with indicated plasmids. Immunoprecipitated DCC, neogenin, or UNC5h2 were probed with the RC20 (Ptyr) and anti-Myc antibodies. Cell lysates were also probed with anti-Flag antibodies to reveal the expression of PYK2. b, c Differential association of $\mathrm{SH} 2$ domain-containing proteins with tyrosine phosphorylated DCC, neogenin, and UNC5h2. HEK 293 cells were cotransfected with Flag-PYK2 and MycDCC, neogenin, or UNC5h2. Cell lysates were mixed with indicated GST-SH2 domain containing fusion proteins $(5 \mu \mathrm{g})$ immobilized on beads. Bound proteins were immunoblotted with anti-Myc and anti-Flag antibodies in (b). Cell lysates for GST pull-down assays were probed with anti-Myc and anti-Flag antibodies in (c).

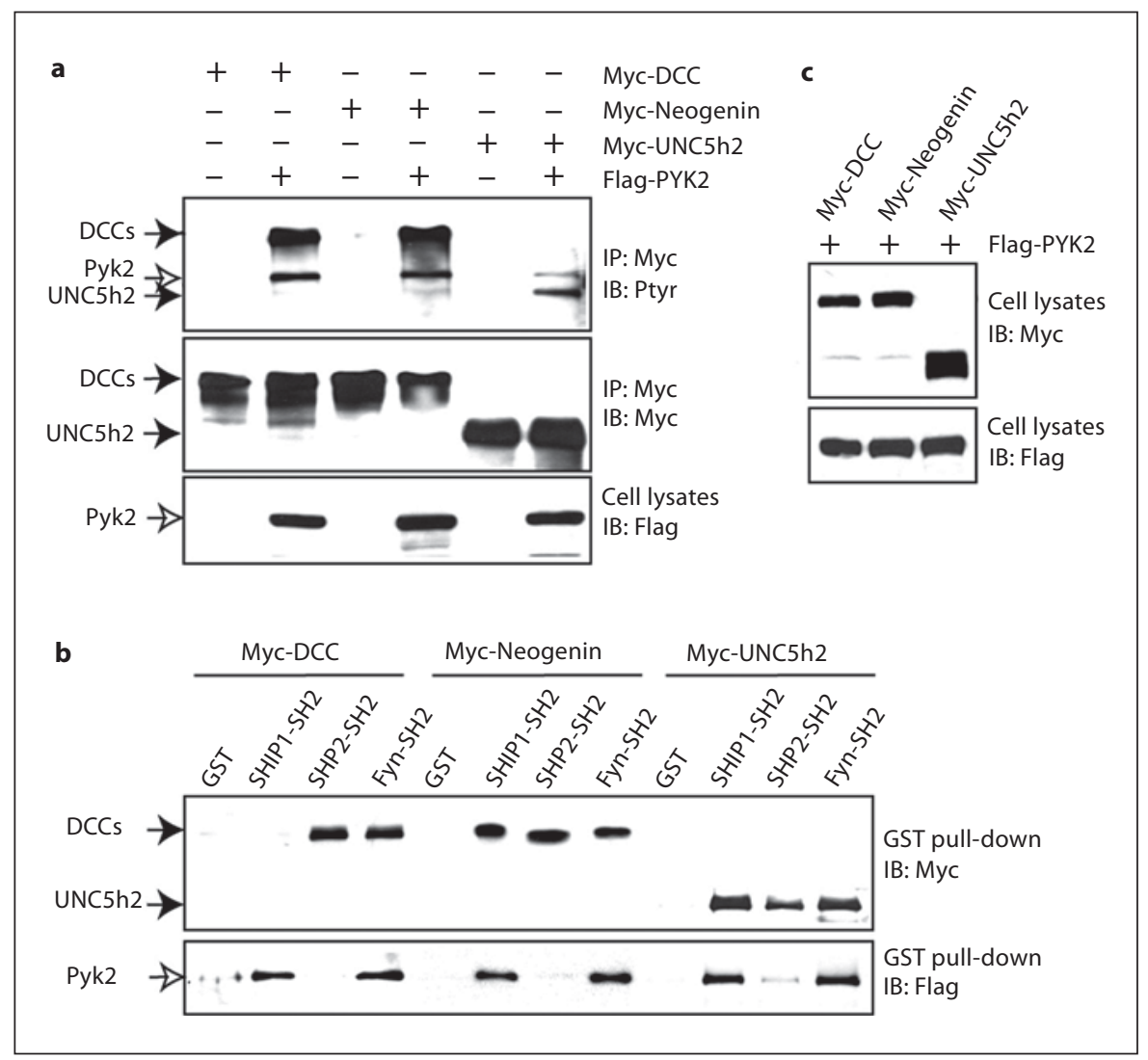

Association of SH2 Domain Containing Proteins with Tyrosine-Phosphorylated DCC, Neogenin, and Unc5H2

Tyrosine phosphorylation often creates docking site(s) for SH2 domain-containing proteins. To identify potential DCC interacting proteins, we incubated lysates containing tyrosine-phosphorylated DCC with recombinant $\mathrm{SH} 2$ domains of various signaling proteins. As shown in figure 4a, DCC was detected in precipitates with the SH2 domains of Fyn and Lck, both Src family tyrosine kinases, and Crk, an adapter protein implicated in regulating cell migration and neurite outgrowth [35]. A modest association was also detected between DCC and the p85 subunit of PI 3-kinase (fig. 4a). However, DCC was not detectable in precipitates with the $\mathrm{SH} 2$ domains of c-Abl, Grb2, Shc, or SHIP (fig. 4a). DCC binding with the Fyn $\mathrm{SH} 2$ domain has the following characteristics. First, it is specific among Src family members. The SH2 domains of Fyn and Lck, but not Src, interacted with DCC (fig. 4a). Second, it required Y1363 and Y1420 phosphorylation of DCC, since the mutation of these residues to phenylalanine blocked or attenuated the binding (fig. 4b).

Tyrosine Phosphorylation of Netrin Receptors
We next compared the binding of several $\mathrm{SH} 2$ domains with DCC, neogenin, and UNC-5. To this end, PYK2 was cotransfected with DCC, neogenin, or Unc$5 \mathrm{H} 2$ in HEK 293 cells for the following reasons. First, coexpression of FAK failed to induce Unc-5H2 tyrosine phosphorylation even in the presence of netrin-1 (data not shown), suggesting little, if any, effect of FAK in netrin-1-induction of Unc-5H2 tyrosine phosphorylation. Second, UNC-5 tyrosine phosphorylation is reported to be induced by co-expression of $\mathrm{v}$-Src [20], and PYK2 is a strong activator of Src kinases (data not shown). Third, in addition to DCC, tyrosine phosphorylation of neogenin and Unc-5H2 was induced upon co-expression of PYK2 in HEK 293 cells (fig. 5a). We then incubated lysates containing tyrosine-phosphorylated DCC, neogenin, or Unc-5H2 with recombinant SH2 domains of Fyn, SHP2, and SHIP. SHP2 is a tyrosine phosphatase that binds to UNC-5 in a SH2 domain-dependent manner [20]. SHIP is a $\mathrm{SH} 2$ domain containing inositol phosphatase frequently associated with ITIM (immune-receptor tyrosine-based inhibitory motif)-containing receptors [36]. While the SH2 domains of Fyn and SHP2 were able to 

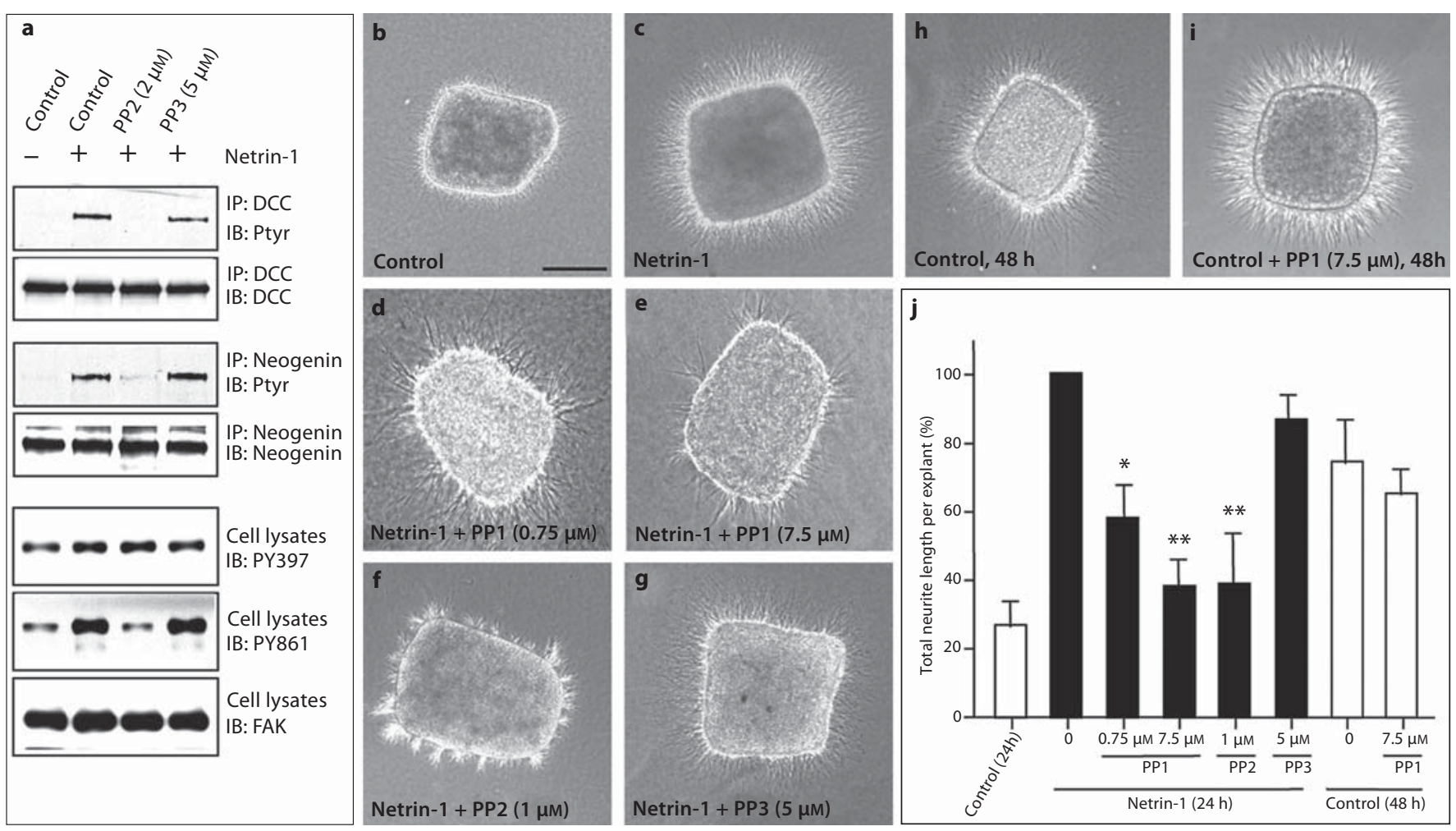

Fig. 6. Requirement of a Src kinase for netrin-1-stimulated protein tyrosine phosphorylation and neurite outgrowth. a Inhibition of netrin-1-induced protein tyrosine phosphorylation of DCC, neogenin, FAK PY861 by PP2, but not PP3. Neurons were pretreated with or without PP2 $(2 \mu \mathrm{M})$ or PP3 $(5 \mu \mathrm{M})$ prior to netrin-1 stimulation. Neuronal lysates were incubated with antiDCC or neogenin antibodies for immunoprecipitation, and the immunoprecipitated proteins were probed with indicated antibodies. For FAK tyrosine phosphorylation, neuronal lysates were subjected to the Western blot analysis using the indicated phosphorylation state-specific antibodies. $\mathbf{b}-\mathbf{j}$ Effects of PP1, PP2, and PP3 on netrin-1-induced and netrin-1-independent cortical neurite outgrowth. Rat cortical explants (E14.5) were cultured in col- lagen gels with control (b), netrin-1 medium (c), or netrin-1 medium containing indicated doses of PP1 (d, e), PP2 (f), or PP3 (g) for $24 \mathrm{~h}$. For netrin-1-independent cortical neurite outgrowth, explants were cultured in collagen gels with control medium in the absence (h) or presence of PP1 $(7.5 \mu \mathrm{M})$ (i) for $48 \mathrm{~h}$. Scale bar $=150$ $\mu \mathrm{m}$. j Quantification of the blocking effects of PP1, PP2, and PP3 on netrin-1-induced and netrin-1-independent cortical neurite outgrowth. The total length of neurites growing into the collagen gels was measured in each explant and normalized to the values obtained from explants cultured in the presence of netrin-1 without inhibitor. Values shown are means $\pm \operatorname{SEM}(n=5) .{ }^{*} \mathrm{p}<0.05$, ${ }^{* *} \mathrm{p}<0.01$, different from netrin-1-stimulated neurite outgrowth (Kolmogorov-Smirnov test). precipitate DCC, neogenin, and Unc-5H2, the $\mathrm{SH} 2$ domain of SHIP only interacted with phosphorylated neogenin and Unc-5H2, but not with DCC (fig. 5c). These results suggest that differential $\mathrm{SH} 2$ domain-containing signaling proteins could be recruited by phosphorylated DCC, neogenin, and Unc-5H2.

\section{Requirement of Src Family Kinases for}

Netrin-1-Stimulated Tyrosine Phosphorylation

of DCC and Neogenin and Neurite Outgrowth

Our results suggest that upon tyrosine phosphorylation, DCC, neogenin, and UNC-5 may interact with sev- eral SH2 domain-containing proteins including Fyn and Lck and implicate that a Src family kinase may be important in mediating and/or regulating netrin-1 function. To test this hypothesis, we first explored the consequence of Src kinase inhibition on netrin-1-induced tyrosine phosphorylation of DCC and neogenin. Pretreatment of neurons with PP2 completely blocked netrin-1-induced tyrosine phosphorylation of DCC and neogenin (fig. 6a). In contrast, PP3, a PP2 chemical homologue with less specificity and potency, had no effect (fig. 6a). The PP2 effect appeared to be specific, since it inhibited netrin-1-induced FAK tyrosine 861, but not 397, phosphorylation 
(fig. 6a), consistent with previous reports [37, 38]. Taken together, these results suggest that an Src family kinase may be involved in netrin-1-induced tyrosine phosphorylation of DCC and neogenin.

We next tested whether inhibition of Src family kinases affects netrin-1-stimulated neurite outgrowth in rat embryonic cortical explants. In the absence of netrin1 , neurites are few and short (fig. 6b). A significant increase in neurite outgrowth (both in number and length) was observed when explants were stimulated with netrin1 (fig. 6c) [33, 39]. Importantly, this event was inhibited by inhibitors of Src family kinases including PP1 and PP2, but not PP3 (fig. 6d-g). Of note is that the inhibitory effect by PP1 appeared to be specific and was not associated with necrosis of the explants even at high concentration $(7.5 \mu \mathrm{M})$ (fig. 6e, j). This was in contrast to PP2, which at high concentration (5 $\mu \mathrm{M}$ or above) may cause cell death (data not shown). In addition, PP1 had no effect on netrin-1-independent neurite outgrowth (fig. 6h-j), suggesting that the observed inhibitory effect by PP1 does not result from a general inhibition of neurite outgrowth, but rather specific inhibition of netrin-1-induced neurite outgrowth. In addition, we noticed that PP1- or PP2-treated explants had a significantly reduced number of neurites and increased thickness of the neurite bundles, implicating a potential role of an Src kinase in neurite fasciculation. Taken together, these results support the notion that an Src kinase may be involved in netrin-1-stimulated cortical neurite outgrowth.

\section{Discussion}

DCC tyrosine phosphorylation has been implicated in netrin-1-mediated axon pathfinding and the involved kinases are beginning to be identified [24-26]. Recent data suggest that FAK may be the tyrosine kinase responsible for netrin-1-induced protein tyrosine phosphorylation [23-25]. Indeed, both FAK and DCC become tyrosinephosphorylated rapidly after netrin-1 stimulation in neurons, apparently prior to neurite outgrowth or turning. Netrin-1-induced DCC tyrosine phosphorylation requires FAK expression [24]. The FAK splice variant, FAK $+6,7$, has been found to be enriched in the developing forebrain [40], implicating a role of this isoform in netrin1 signaling. In addition to FAK, an Src family kinase appears to be involved in netrin-1 signaling $[23,25]$. Our results that Src kinase inhibitors block netrin-1-induced DCC tyrosine phosphorylation, neurite outgrowth, and attractive response to TAG-1-positive neuritis support this idea. The Src family has multiple members including c-Src, Fyn, Lck, and Lyn. Fyn, but not Src, may participate in netrin-1 signaling for the following reasons. First, PP2, a Src family kinase inhibitor that displays a higher affinity for Fyn than Src [41], suppressed netrin-1-induced DCC tyrosine phosphorylation. Second, the $\mathrm{SH} 2$ domain of Fyn, but not that of Src, associates with tyrosine-phosphorylated DCC in vitro. Finally, our results provide a working model for Fyn activation in the netrin-1 signaling cascade. Netrin-1 activates FAK [23-25] and induces the formation of a protein complex centered around DCC/FAK including an Src family kinase (likely Fyn) that leads to the increased tyrosine phosphorylation of DCC. Phosphorylation of DCC in turn facilitates the DCC-Fyn interaction, forming a positive reinforcement cycle. This protein complex is required for netrin-1stimulated axon outgrowth and attractive growth cone turning.

Our results suggest a different mechanism involved in netrin-1-induced DCC and neogenin tyrosine phosphorylation. While netrin-1 is sufficient to induce DCC tyrosine phosphorylation in cells coexpressing FAK (e.g. HEK293 cells) or in cells containing high levels of endogenous FAK (e.g. in neurons), netrin-1 fails to increase neogenin tyrosine phosphorylation in non-neuronal cells even in the presence of high level of FAK. These results suggest that neogenin may need a 'coreceptor' to be stimulated by netrin-1. This coreceptor may be present in neurons, but not in HEK 293 or fibroblasts. Interestingly, a recent publication suggests that $\mathrm{CDO}$, an immunoglobin superfamily receptor that is specifically expressed in myoblasts, appears to be a co-receptor of neogenin in $\mathrm{C} 2 \mathrm{C} 12$ cells, and the neogenin-CDO receptor complexes are required for netrin-3-induced myotube formation [42].

DCC and neogenin appear to mediate different responses during axon pathfinding. DCC is involved in the netrin-1-induced attractive response while neogenin may function as a RGM receptor to mediate a repulsive response [16, 17]. Exactly how tyrosine phosphorylation of the two proteins regulates netrin-1 signaling and function remains unclear. One possibility, supported by data presented in this paper, is that DCC tyrosine phosphorylation may increase the local concentration of effector molecules including Fyn, Lck, or Crk. Recruitment of these proteins into the DCC complex may be important for netrin-1 activation of downstream signaling that leads to neurite outgrowth and attractive growth cone turning. This possibility is in agreement with recent publications that Fyn is involved in netrin-1 signaling and functions 
$[23,25,26]$. Crk is a SH2 domain containing adaptor protein that binds to p130Cas and is implicated in integrinmediated cell migration [43,44]. Our data showing that tyrosine-phosphorylated DCC binds to Crk provide new leads to netrin-1 signaling and further suggest a similarity of signaling events triggered by the engagement of integrin and DCC. The exact role of neogenin tyrosine phosphorylation in netrin-1 signaling and function remains largely unclear. We propose that tyrosine phosphorylation of DCC and neogenin may promote signal discrimination. The tyrosine phosphorylation sites in DCC and neogenin are not identical, and hence may be able to associate with distinct molecules or associate with the same effectors with different affinities. Alternatively, neogenin or tyrosine-phosphorylated neogenin could act to sequester effector molecules (e.g. FAK) in their inactive forms, making such molecules unavailable to DCC receptors. This is supported by the finding that neogeninassociated FAK appears to be the unphosphorylated 'inactive' form (data not shown). Taken together, our results suggest differential mechanisms of DCC and neogenin in response to netrin-1 and in mediating downstream signaling events.

\section{Acknowlegements}

We are grateful to Drs. Y. Rao (Washington University), J.Y. $\mathrm{Wu}$ (Washington University), and B. Vogelstein (Johns Hopkins Medical School) for reagents. This study is supported by grants from NIH to L.M. and W.-C.X.

\section{References}

1 Livesey FJ: Netrins and netrin receptors. Cell Mol Life Sci 1999;56:62-68.

2 Colavita A, Culotti JG: Suppressors of ectopic UNC-5 growth cone steering identify eight genes involved in axon guidance in Caenorhabditis elegans. Dev Biol 1998;194: 72-85.

-3 Kennedy TE, Serafini T, de la Torre JR, Tessier-Lavigne M: Netrins are diffusible chemotropic factors for commissural axons in the embryonic spinal cord. Cell 1994;78: 425-435.

-4 Serafini T, Kennedy TE, Galko MJ, Mirzayan C, Jessell TM, Tessier-Lavigne M: The netrins define a family of axon outgrowth-promoting proteins homologous to C. elegans UNC-6. Cell 1994;78:409-424.

5 Colamarino SA, Tessier-Lavigne M: The axonal chemoattractant netrin-1 is also a chemorepellent for trochlear motor axons. Cell 1995;81:621-629.

6 Keino-Masu K, Masu M, Hinck L, Leonardo ED, Chan SS, Culotti JG, Tessier-Lavigne M: Deleted in colorectal cancer (DCC) encodes a netrin receptor. Cell 1996;87:175-185.

7 Chan SS, Zheng H, Su MW, Wilk R, Killeen MT, Hedgecock EM, Culotti JG: UNC-40, a C. elegans homolog of DCC (deleted in colorectal cancer), is required in motile cells responding to UNC-6 netrin cues. Cell 1996; 87:187-195.

-8 Kolodziej PA, Timpe LC, Mitchell KJ, Fried SR, Goodman CS, Jan LY, Jan YN: Frazzled encodes a Drosophila member of the DCC immunoglobulin subfamily and is required for CNS and motor axon guidance. Cell 1996;87:197-204.
Hiramoto M, Hiromi Y, Giniger E, Hotta Y: The Drosophila netrin receptor Frazzled guides axons by controlling netrin distribution. Nature 2000;406:886-889.

10 Culotti JG, Merz DC: DCC and netrins. Curr Opin Cell Biol 1998;10:609-613.

11 Hedgecock EM, Culotti JG, Hall DH: The unc-5, unc- 6 , and unc- 40 genes guide circumferential migrations of pioneer axons and mesodermal cells on the epidermis in $C$. elegans. Neuron 1990;4:61-85.

12 Hong K, Hinck L, Nishiyama M, Poo MM, Tessier-Lavigne M, Stein E: A ligand-gated association between cytoplasmic domains of UNC5 and DCC family receptors converts netrin-induced growth cone attraction to repulsion. Cell 1999;97:927-941.

13 Keleman K, Dickson BJ: Short- and longrange repulsion by the Drosophila Unc5 netrin receptor. Neuron 2001;32:605-617.

14 Ackerman SL, Kozak LP, Przyborski SA, Rund LA, Boyer BB, Knowles BB: The mouse rostral cerebellar malformation gene encodes an UNC-5-like protein. Nature 1997; 386:838-842.

15 Leonardo ED, Hinck L, Masu M, KeinoMasu K, Ackerman SL, Tessier-Lavigne M: Vertebrate homologues of $C$. elegans UNC-5 are candidate netrin receptors. Nature 1997; 386:833-838.

16 Rajagopalan S, Deitinghoff L, Davis D, Conrad S, Skutella T, Chedotal A, Mueller BK, Strittmatter SM: Neogenin mediates the action of repulsive guidance molecule. Nat Cell Biol 2004;6:756-762.

$\checkmark 17$ Matsunaga E, Tauszig-Delamasure S, Monnier PP, Mueller BK, Strittmatter SM, Mehlen P, Chedotal A: RGM and its receptor neogenin regulate neuronal survival. Nat Cell Biol 2004;6:749-755.
18 Flanagan JG, Vanderhaeghen P: The ephrins and Eph receptors in neural development. Annu Rev Neurosci 1998;21:309-345.

19 Bashaw GJ, Kidd T, Murray D, Pawson T, Goodman CS: Repulsive axon guidance: Abelson and Enabled play opposing roles downstream of the roundabout receptor. Cell 2000;101:703-715

20 Tong J, Killeen M, Steven R, Binns KL, Culotti J, Pawson T: Netrin stimulates tyrosine phosphorylation of the UNC-5 family of netrin receptors and induces Shp2 binding to the RCM cytodomain. J Biol Chem 2001;276: 40917-40925

21 Killeen M, Tong J, Krizus A, Steven R, Scott I, Pawson T, Culotti J: UNC-5 function requires phosphorylation of cytoplasmic tyrosine 482, but its UNC-40-independent functions also require a region between the $\mathrm{ZU}-5$ and death domains. Dev Biol 2002;251:348366.

22 Chang C, Yu TW, Bargmann CI, TessierLavigne M: Inhibition of netrin-mediated axon attraction by a receptor protein tyrosine phosphatase. Science 2004;305:103106.

23 Liu G, Beggs H, Jurgensen C, Park HT, Tang H, Gorski J, Jones KR, Reichardt LF, Wu J, Rao Y: Netrin requires focal adhesion kinase and Src family kinases for axon outgrowth and attraction. Nat Neurosci 2004;7:12221232

24 Ren XR, Ming GL, Xie Y, Hong Y, Sun DM, Zhao ZQ, Feng Z, Wang Q, Shim S, Chen ZF, Song HJ, Mei L, Xiong WC: Focal adhesion kinase in netrin-1 signaling. Nat Neurosci 2004;7:1204-1212. 
>25 Li W, Lee J, Vikis HG, Lee SH, Liu G, Aurandt J, Shen TL, Fearon ER, Guan JL, Han M, Rao Y, Hong K, Guan KL: Activation of FAK and Src are receptor-proximal events required for netrin signaling. Nat Neurosci 2004; 7:1213-1221.

-26 Meriane M, Tcherkezian J, Webber CA, Danek EI, Triki I, McFarlane S, Bloch-Gallego E, Lamarche-Vane N: Phosphorylation of DCC by Fyn mediates netrin-1 signaling in growth cone guidance. J Cell Biol 2004; 167:687-698.

-27 Li HS, Chen JH, Wu W, Fagaly T, Zhou L, Yuan W, Dupuis S, Jiang ZH, Nash W, Gick C, Ornitz DM, Wu JY, Rao Y: Vertebrate slit, a secreted ligand for the transmembrane protein roundabout, is a repellent for olfactory bulb axons. Cell 1999;96:807-818.

-28 Tang H, Kerins DM, Hao Q, Inagami T, Vaughan DE: The urokinase-type plasminogen activator receptor mediates tyrosine phosphorylation of focal adhesion proteins and activation of mitogen-activated protein kinase in cultured endothelial cells. J Biol Chem 1998;273:18268-18272.

-29 Xiong W, Parsons JT: Induction of apoptosis after expression of PYK2, a tyrosine kinase structurally related to focal adhesion kinase. J Cell Biol 1997;139:529-539.

-30 Ren XR, Du QS, Huang YZ, Ao SZ, Mei L, Xiong WC: Regulation of CDC42 GTPase by proline-rich tyrosine kinase 2 interacting with PSGAP, a novel pleckstrin homology and Src homology 3 domain containing rhoGAP protein. J Cell Biol 2001;152:971984
31 Huang YZ, Won S, Ali DW, Wang Q, Tanowitz M, Du QS, Pelkey KA, Yang DJ, Xiong WC, Salter MW, Mei L: Regulation of neuregulin signaling by PSD-95 interacting with ErbB4 at CNS synapses. Neuron 2000;26: 443-455.

32 Wong K, Ren XR, Huang YZ, Xie Y, Liu G, Saito H, Tang H, Wen L, Brady-Kalnay SM, Mei L, Wu JY, Xiong WC, Rao Y: Signal transduction in neuronal migration: roles of GTPase activating proteins and the small GTPase Cdc42 in the Slit-Robo pathway. Cell 2001;107:209-221.

33 Metin C, Deleglise D, Serafini T, Kennedy TE, Tessier-Lavigne M: A role for netrin-1 in the guidance of cortical efferents. Development 1997;124:5063-5074.

34 Braisted JE, Catalano SM, Stimac R, Kennedy TE, Tessier-Lavigne M, Shatz CJ, O'Leary DD: Netrin-1 promotes thalamic axon growth and is required for proper development of the thalamocortical projection. J Neurosci 2000;20:5792-5801.

35 Yang LT, Alexandropoulos K, Sap J: c-SRC mediates neurite outgrowth through recruitment of Crk to the scaffolding protein Sin/Efs without altering the kinetics of ERK activation. J Biol Chem 2002;277:1740617414.

36 Sly LM, Rauh MJ, Kalesnikoff J, Buchse T, Krystal G: SHIP, SHIP2, and PTEN activities are regulated in vivo by modulation of their protein levels: SHIP is up-regulated in macrophages and mast cells by lipopolysaccharide. Exp Hematol 2003;31:1170-1181.

37 Abu-Ghazaleh R, Kabir J, Jia H, Lobo M Zachary I: Src mediates stimulation by vascular endothelial growth factor of the phosphorylation of focal adhesion kinase at tyrosine 861, and migration and anti-apoptosis in endothelial cells. Biochem J 2001;360: 255-264.
38 Calalb MB, Zhang X, Polte TR, Hanks SK: Focal adhesion kinase tyrosine-861 is a major site of phosphorylation by Src. Biochem Biophys Res Commun 1996;228:662-668.

39 Richards LJ, Koester SE, Tuttle R, O’Leary DD: Directed growth of early cortical axons is influenced by a chemoattractant released from an intermediate target. J Neurosci 1997; 17:2445-2458.

40 Toutant M, Studler JM, Burgaya F, Costa A, Ezan P, Gelman M, Girault JA: Autophosphorylation of Tyr397 and its phosphorylation by Src-family kinases are altered in focal-adhesion-kinase neuronal isoforms. Biochem J 2000;348:119-128.

-41 Derkinderen P, Toutant M, Kadare G, Ledent C, Parmentier M, Girault JA: Dual role of Fyn in the regulation of FAK $+6,7$ by cannabinoids in hippocampus. J Biol Chem 2001; 276:38289-38296.

42 Kang JS, Yi MJ, Zhang W, Feinleib JL, Cole F, Krauss RS: Netrins and neogenin promote myotube formation. J Cell Biol 2004;167: 493-504.

43 Polte TR, Hanks SK: Complexes of focal adhesion kinase (FAK) and Crk-associated substrate (p130(Cas)) are elevated in cytoskeleton-associated fractions following adhesion and Srctransformation: requirements for Src kinase activity and FAK proline-rich motifs. J Biol Chem 1997;272:5501-5509.

44 Feller SM: Crk family adaptors-signalling complex formation and biological roles. Oncogene 2001;20:6348-6371. 